\title{
Dimensões dos ensaios e estimativas do tamanho ótimo de parcela em batata
}

\author{
Lindolfo Storck ${ }^{(1)}$, Dílson Antônio Bisognin ${ }^{(1)}$ e Sérgio José Ribeiro de Oliveira ${ }^{(2)}$
}

\begin{abstract}
(1)Universidade Federal de Santa Maria, Dep. de Fitotecnia, Av. Roraima s/no, Bairro Camobi, CEP 97105-900 Santa Maria, RS. E-mail: lindolfo@smail.ufsm.br, dilsonb@smail.ufsm.br (2)Universidade Estadual de Santa Cruz, Dep. de Ciências Exatas e Tecnológicas, Rod. Ilhéus-Itabuna, Km 16, CEP 45650-000 Ilhéus, BA. E-mail: solive2002@uol.com.br
\end{abstract}

Resumo - O objetivo deste trabalho foi estimar a relação entre dimensões da área dos ensaios em branco, as estimativas do tamanho ótimo de parcela e a precisão de experimentos com a cultura da batata. O ensaio de uniformidade foi conduzido na área de produção de batata, da Fundação Estadual de Pesquisa Agropecuária, em Júlio de Castilhos, RS. A produtividade de tubérculos de batata em 3.456 covas (24 linhas de 144 covas) foi usada para planejar dez ensaios com variações no número de linhas (largura) e de covas por linha (comprimento). Em cada ensaio, foram estimados o tamanho ótimo de parcela e a diferença entre médias de tratamentos. As análises de correlação linear e de trilha permitiram verificar que o tamanho dos ensaios com a cultura da batata, medido em número de covas, não influencia a estimativa do índice de heterogeneidade do solo e a estimativa do tamanho ótimo de parcela. Áreas experimentais com maior número de covas por linha permitem planejar experimentos mais precisos quanto à avaliação da produtividade de tubérculos de batata. Parcelas com seis covas na linha e a adoção da linha como bloco aumentam a precisão dos experimentos com batata.

Termos para indexação: Solanum tuberosum, planejamento de experimento, tamanho de parcela; área experimental limitada.

\section{Potato trial dimensions and optimum plot size estimations}

\begin{abstract}
The objective of this work was to assess the relationship among experimental area in uniformity trial dimensions, optimum plot size estimations and experimental precision of potato trials. A homogeneity trial was carried out at the production site of Fundação Estadual de Pesquisa Agropecuária, Júlio de Castilhos, RS, Brazil. Tuber yield of 3,456 hills (24 rows of 144 hills) was quantified and data were used for planning ten different size trials, in which the number of rows and hills per row varied. Estimations of optimum plot size and differences between means of treatments were done for each trial. Based on linear correlation and path analysis, the size of potato trial using different hill numbers does not affect the estimation of soil heterogeneity index and the estimation of optimum plot size. Experimental areas with high number of hills per row can be used to improve precision of potato yield evaluations. One row with six hill plots and treatments distributed in the row, taken as block, increase experimental precision in potato.
\end{abstract}

Index terms: Solanum tuberosum, experimental planning, plot size, limited experimental area.

\section{Introdução}

De modo a diminuir o erro experimental, requisitos como número de repetições, seleção dos tratamentos, heterogeneidade do material experimental, diferença no número de plantas dentro da parcela, tipo de tratamento aplicado, tratos culturais, manejo e controle de pragas, doenças e plantas daninhas, amostragem na parcela e, principalmente, heterogeneidade das unidades experimentais devem ser atendidos (Gomez \& Gomez, 1984; Lopes et al., 1994; Lopes \& Storck, 1995; Steel et al., 1997; Ramalho et al., 2000; Storck et al., 2000).
Ao planejar um experimento, deve-se considerar que os recursos materiais, financeiros e humanos são, geralmente, restritos. Por isso, além de se buscar resultados confiáveis, deve-se garantir experimentos e dados precisos. Ao enfatizar as formas de controle do erro experimental gerado pela heterogeneidade das unidades experimentais, deve-se dedicar atenção a algumas práticas, tais como a execução de experimento em branco, para adequar a área experimental escolhida ao delineamento, ao tamanho e forma de parcela, ao número de repetições e de tratamentos com a precisão desejada. Estas combinações consistem no principal modo de 
se contornar a heterogeneidade da produtividade, buscando maximizar as informações obtidas na área experimental disponível (Storck et al., 2000).

Existem vários métodos para estimar o tamanho ótimo de parcela, que consideram diversos fatores nas determinações (Bakke, 1988). O método de Smith (1938) relaciona o tamanho ótimo de parcela com o índice de heterogeneidade do solo, e o método de Hatheway (1961), com o número de repetições e a precisão desejada. Métodos como o de Lessman \& Atkins (1963) e Meier \& Lessman (1971) relacionam o tamanho ótimo de parcela com as estimativas dos parâmetros da função $\mathrm{CV}(\mathrm{x})=\mathrm{A} / \mathrm{X}^{\mathrm{B}}$ e os descritos por Thomas (1974), com as funções $\mathrm{VU}(\mathrm{x})=\mathrm{V}_{1} / \mathrm{X}^{\mathrm{b}}$ e $\mathrm{CV}(\mathrm{x})=\mathrm{V}_{1} /\left[\mathrm{M}_{1}\left(\mathrm{X}^{\mathrm{b}}\right)^{1 / 2}\right]$. Apesar dessa variedade de métodos e aplicações a diversas culturas, não foram encontrados trabalhos que relacionam a área do ensaio com as estimativas do tamanho ótimo de parcela e com as estimativas dos parâmetros $\mathrm{V}_{1}, \mathrm{~b}$, A e $\mathrm{B}$ das funções $\mathrm{VU}(\mathrm{x})=\mathrm{V}_{1} / \mathrm{X}^{\mathrm{b}}$ e $\mathrm{CV}(\mathrm{x})=\mathrm{A} / \mathrm{X}^{\mathrm{B}}$. Além disso, existem poucas aplicações que se referem à utilização de áreas experimentais limitadas (Ramalho et al., 2000; Storck et al., 2000) como fator relevante no planejamento e condução de experimentos. Em muitos casos, a área experimental é predeterminada e o ensaio necessita ser readequado.

O objetivo deste trabalho foi estimar a relação entre as dimensões dos ensaios e as estimativas do tamanho ótimo de parcela e a precisão de experimentos com a cultura da batata.

\section{Material e Métodos}

Um ensaio de uniformidade foi conduzido na área de produção de batata da Fundação Estadual de Pesquisa Agropecuária, em Júlio de Castilhos. A área recebeu tratos culturais e fitossanitários uniformes (Bisognin, 1996) e a colheita foi realizada nos dias 28/11 e 1\%/12 de 2003. Foi avaliada a produtividade de tubérculos de batata da cultivar Macaca em 3.456 covas distribuídas em 24 linhas (largura) de 144 covas (comprimento). $\mathrm{O}$ espaçamento entre linhas foi de $0,80 \mathrm{~m}$ e entre covas de $0,30 \mathrm{~m}$.

As 3.456 covas, ou unidades básicas (UB), foram usadas para planejar dez casos de ensaios com diferentes subáreas e formas, variando o número de linhas ou largura (L) e o número de covas por linha ou comprimento (C). O número de ensaios (número de repetições) de cada caso foi igual a 3.456 covas dividido pelo número de covas de cada caso (Tabela 1). Cada caso foi repetido de acordo com a divisão da área total; por exemplo, no caso 2, a área foi dividida em duas, cada uma com 1.728 UB. Em cada caso e ensaio, foram planejadas parcelas de diferentes tamanhos e formas, cujas dimensões formam submúltiplos das dimensões de cada subárea (ensaio), com $(\mathrm{L} \leq \mathrm{C})$, buscando o aproveitamento total de cada área experimental, considerando que o número de linhas de cada parcela foi $\mathrm{L} \leq 6$, e o de colunas $\mathrm{C} \leq 12$. Portanto, os tamanhos de parcelas foram simulados pelo agrupamento de unidades contíguas, de modo que LxC correspondesse a X, o tamanho de parcela, em unidades básicas.

Em cada caso e ensaio, foram determinados: $\mathrm{N}$ - número de parcelas com X UB de tamanho; $\mathrm{V}(\mathrm{x})$ - variância entre as parcelas de X UB de tamanho; VU(x) - variância por UB para parcelas de X UB, $\mathrm{VU}(\mathrm{x})=\mathrm{V}(\mathrm{x}) / \mathrm{X}^{2} ; \mathrm{CV}(\mathrm{x})$ - coeficiente de variação entre as parcelas de $\mathrm{X}$ UB de tamanho; e, $\mathrm{M}(\mathrm{x})$ igual à média das parcelas de X UB de tamanho, e $\mathrm{M}_{1}$ - média das parcelas de uma UB. Com base nessas determinações estimou-se, em cada caso e ensaio, o índice de heterogeneidade do solo (b), usando a função $\mathrm{VU}(\mathrm{x})=\mathrm{V}_{1} / \mathrm{X}^{\mathrm{b}}$ de Smith (1938). Os parâmetros $\mathrm{V}_{1} \mathrm{e}$ b foram estimados mediante a transformação logarítmica da função e da estimação ponderada pelos números de graus de liberdade (Steel et al., 1997). De forma semelhante, estimaram-se os parâmetros da função $\mathrm{CV}(\mathrm{x})=\mathrm{A} / \mathrm{X}^{\mathrm{B}}$. Também foram calculados os coeficientes de determinação $\left(\mathrm{R}^{2}\right)$ e as estimativas de tamanhos ótimos de parcelas $\left(\mathrm{X}_{0}\right)$ pelo método da máxima curvatura modificada de Meier \& Lessman (1971), a partir da seguinte expressão:

$\mathrm{X}_{0}=\exp \left\{\left(\frac{1}{2 \mathrm{~B}+2}\right) \ln \left[\frac{\mathrm{A}^{2} \mathrm{~B}^{2}(2 \mathrm{~B}+1)}{\mathrm{B}+2}\right]\right\}$.

A diferença verdadeira entre médias de tratamentos, expressa em porcentagem da média (D), foi estimada pelo método de Hatheway (1961), cuja fórmula é $\mathrm{D}=\sqrt{2\left(\mathrm{t}_{1}+\mathrm{t}_{2}\right)^{2} \mathrm{~A}^{2} / \mathrm{rX}_{0}^{\mathrm{b}}}$, em que r é o número de repetições; $\mathrm{X}_{0}$ é o tamanho ótimo de parcelas, em números de UB; A é a estimativa do coeficiente de variação com uma UB; b é a estimativa do índice de heterogeneidade do solo; $\mathrm{t}_{1}$ é o valor crítico da distribuição de t para testes de significância (bilateral a 5\%); e $\mathrm{t}_{2}$ é o valor crítico da distribuição de t, correspondente a um erro de 2(1 - P), sendo $\mathrm{P}$ a probabilidade de se obter resultados significativos $(0,80)$. 
Os valores críticos da distribuição de t foram obtidos em função dos números de graus de liberdade, conside- rando o delineamento inteiramente casualizado, ou seja, $\mathrm{GL}=\mathrm{I}(\mathrm{r}-1)$. O número de tratamentos (I) foi arbitrado

Tabela 1. Estimativas do índice de heterogeneidade do solo (b), dos parâmetros da função $C V(x)=A / X^{B}$, do coeficiente de determinação $\left(\mathrm{R}^{2}\right)$, da média de produtividade de tubérculos de batata (kg por cova) nas parcelas de uma unidade básica $\left(\mathrm{M}_{1}\right)$ e do tamanho ótimo de parcelas $\left(\mathrm{X}_{0}\right)$ obtido pelo método da máxima curvatura modificada de Meier \& Lessman e valores médios para os casos com diferentes dimensões de linhas ou larguras (L) e de colunas ou comprimentos (C) e número de unidades básicas (NUB).

\begin{tabular}{|c|c|c|c|c|c|c|c|c|c|c|}
\hline Caso & Ensaio & $\mathrm{L}$ & $\mathrm{C}$ & NUB & $\mathrm{b}$ & $\mathrm{A}$ & $\mathrm{B}$ & $\mathrm{R}^{2}$ & $\mathrm{M}_{1}$ & $\mathrm{X}_{0}$ \\
\hline 1 & 1 & 24 & 144 & 3.456 & 0,3934 & 31,09 & 0,1966 & 0,810 & 0,8466 & 3,75 \\
\hline 2 & 1 & 24 & 72 & 1.728 & 0,4753 & 37,32 & 0,2377 & 0,812 & 0,8557 & 4,93 \\
\hline 2 & 2 & 24 & 72 & 1.728 & 0,2711 & 24,07 & 0,1356 & 0,730 & 0,8374 & 2,25 \\
\hline Média & - & - & - & - & 0,3732 & 30,70 & 0,1866 & 0,771 & 0,8466 & 3,59 \\
\hline 3 & 1 & 12 & 144 & 1.728 & 0,9668 & 45,13 & 0,4835 & 0,629 & 0,9276 & 7,38 \\
\hline 3 & 2 & 12 & 144 & 1.728 & 0,4620 & 34,25 & 0,2311 & 0,770 & 0,7656 & 4,52 \\
\hline Média & - & - & - & - & 0,7144 & 39,69 & 0,3573 & 0,700 & 0,8466 & 5,95 \\
\hline 4 & 1 & 24 & 48 & 1.152 & 0,6671 & 42,21 & 0,3335 & 0,752 & 0,8871 & 6,40 \\
\hline 4 & 2 & 24 & 48 & 1.152 & 0,3286 & 33,89 & 0,1644 & 0,830 & 0,7950 & 3,54 \\
\hline 4 & 3 & 24 & 48 & 1.152 & 0,4073 & 24,03 & 0,2036 & 0,776 & 0,8577 & 3,10 \\
\hline Média & - & - & - & - & 0,4677 & 33,37 & 0,2338 & 0,786 & 0,8466 & 4,35 \\
\hline 5 & 1 & 24 & 36 & 864 & 0,6377 & 39,49 & 0,3188 & 0,645 & 0,8928 & 5,98 \\
\hline 5 & 2 & 24 & 36 & 864 & 0,3379 & 34,02 & 0,1690 & 0,828 & 0,8187 & 3,63 \\
\hline 5 & 3 & 24 & 36 & 864 & 0,2895 & 28,53 & 0,1448 & 0,819 & 0,8045 & 2,76 \\
\hline 5 & 4 & 24 & 36 & 864 & 0,5003 & 25,23 & 0,2500 & 0,786 & 0,8704 & 3,71 \\
\hline Média & - & - & - & - & 0,4414 & 31,81 & 0,2207 & 0,770 & 0,8466 & 4,02 \\
\hline 6 & 1 & 12 & 72 & 864 & 0,9657 & 53,47 & 0,4826 & 0,585 & 0,9341 & 8,28 \\
\hline 6 & 2 & 12 & 72 & 864 & 0,4555 & 36,49 & 0,2278 & 0,637 & 0,7774 & 4,72 \\
\hline 6 & 3 & 12 & 72 & 864 & 0,9541 & 33,91 & 0,4771 & 0,717 & 0,9211 & 6,07 \\
\hline 6 & 4 & 12 & 72 & 864 & 0,4510 & 30,75 & 0,2254 & 0,838 & 0,7537 & 4,08 \\
\hline Média & - & - & - & - & 0,7066 & 38,66 & 0,3532 & 0,694 & 0,8466 & 5,78 \\
\hline 7 & 1 & 12 & 48 & 576 & 0,7289 & 42,86 & 0,3645 & 0,406 & 0,9392 & 6,68 \\
\hline 7 & 2 & 12 & 48 & 576 & 0,6805 & 39,44 & 0,3402 & 0,545 & 0,8350 & 6,13 \\
\hline 7 & 3 & 12 & 48 & 576 & 0,9814 & 39,83 & 0,4910 & 0,489 & 0,9211 & 6,80 \\
\hline 7 & 4 & 12 & 48 & 576 & 0,6681 & 44,46 & 0,3340 & 0,805 & 0,6689 & 6,66 \\
\hline 7 & 5 & 12 & 48 & 576 & 0,6775 & 24,89 & 0,3386 & 0,632 & 0,9227 & 4,34 \\
\hline 7 & 6 & 12 & 48 & 576 & 0,5998 & 28,76 & 0,2999 & 0,763 & 0,7928 & 4,56 \\
\hline Média & - & - & - & - & 0,7227 & 36,71 & 0,3613 & 0,607 & 0,8466 & 5,86 \\
\hline 8 & 1 & 24 & 24 & 576 & 0,7700 & 44,35 & 0,3851 & 0,612 & 0,9094 & 6,97 \\
\hline 8 & 2 & 24 & 24 & 576 & 0,4708 & 35,35 & 0,2354 & 0,583 & 0,8647 & 4,69 \\
\hline 8 & 3 & 24 & 24 & 576 & 0,3025 & 33,22 & 0,1512 & 0,441 & 0,7931 & 3,27 \\
\hline 8 & 4 & 24 & 24 & 576 & 0,2928 & 31,21 & 0,1452 & 0,743 & 0,7969 & 2,99 \\
\hline 8 & 5 & 24 & 24 & 576 & 0,3930 & 25,35 & 0,1965 & 0,844 & 0,8465 & 3,16 \\
\hline 8 & 6 & 24 & 24 & 576 & 0,5021 & 24,87 & 0,2511 & 0,627 & 0,8689 & 3,68 \\
\hline Média & - & - & - & - & 0,4552 & 32,39 & 0,2274 & 0,642 & 0,8466 & 4,13 \\
\hline 9 & 1 & 12 & 36 & 432 & 1,1517 & 69,96 & 0,5758 & 0,607 & 0,9233 & 9,86 \\
\hline 9 & 2 & 12 & 36 & 432 & 0,8093 & 42,53 & 0,4056 & 0,454 & 0,8623 & 6,85 \\
\hline 9 & 3 & 12 & 36 & 432 & 0,9428 & 40,34 & 0,4711 & 0,736 & 0,9449 & 6,82 \\
\hline 9 & 4 & 12 & 36 & 432 & 0,8398 & 50,87 & 0,4203 & 0,767 & 0,6924 & 7,84 \\
\hline 9 & 5 & 12 & 36 & 432 & 1,2870 & 41,65 & 0,6434 & 0,803 & 0,9188 & 7,07 \\
\hline 9 & 6 & 12 & 36 & 432 & 0,6731 & 40,84 & 0,3365 & 0,775 & 0,6902 & 6,27 \\
\hline 9 & 7 & 12 & 36 & 432 & 0,8034 & 29,70 & 0,4018 & 0,814 & 0,9235 & 5,29 \\
\hline 9 & 8 & 12 & 36 & 432 & 0,8344 & 34,16 & 0,4170 & 0,782 & 0,8172 & 5,91 \\
\hline Média & - & - & - & - & 0,9177 & 43,76 & 0,4589 & 0,717 & 0,8466 & 6,99 \\
\hline 10 & 1 & 12 & 24 & 288 & 0,5175 & 38,97 & 0,2587 & 0,434 & 0,9206 & 5,35 \\
\hline 10 & 2 & 12 & 24 & 288 & 0,2963 & 26,75 & 0,1482 & 0,702 & 0,9577 & 2,66 \\
\hline 10 & 3 & 12 & 24 & 288 & 0,4614 & 27,84 & 0,2307 & 0,758 & 0,9240 & 3,82 \\
\hline 10 & 4 & 12 & 24 & 288 & 0,6501 & 24,43 & 0,3248 & 0,531 & 0,9181 & 4,19 \\
\hline 10 & 5 & 12 & 24 & 288 & 0,5353 & 22,24 & 0,2678 & 0,961 & 0,9285 & 3,50 \\
\hline 10 & 6 & 12 & 24 & 288 & 0,4724 & 20,96 & 0,2361 & 0,875 & 0,9169 & 3,08 \\
\hline 10 & 7 & 12 & 24 & 288 & 0,8989 & 44,49 & 0,4496 & 0,417 & 0,8982 & 7,23 \\
\hline 10 & 8 & 12 & 24 & 288 & 1,0961 & 53,85 & 0,5481 & 0,471 & 0,7718 & 8,36 \\
\hline 10 & 9 & 12 & 24 & 288 & 0,7755 & 49,76 & 0,3878 & 0,766 & 0,6621 & 7,58 \\
\hline 10 & 10 & 12 & 24 & 288 & 0,4852 & 38,67 & 0,2445 & 0,697 & 0,6757 & 5,15 \\
\hline 10 & 11 & 12 & 24 & 288 & 0,4698 & 25,51 & 0,2349 & 0,841 & 0,8209 & 3,59 \\
\hline 10 & 12 & 12 & 24 & 288 & 0,6273 & 29,18 & 0,3135 & 0,894 & 0,7646 & 4,72 \\
\hline Média & - & - & - & - & 0,6071 & 33,55 & 0,3037 & 0,696 & 0,8466 & 4,94 \\
\hline
\end{tabular}


em 12 e o número de repetições (r) foi calculado pela divisão do número de covas, de cada tamanho de ensaio, pelo produto do número de tratamentos com o tamanho ótimo de parcela, aproximado para o valor inteiro.

Procedeu-se à análise de correlação linear simples entre as estimativas dos parâmetros da função $\mathrm{CV}(\mathrm{x})=\mathrm{A} / \mathrm{X}^{\mathrm{B}}$, coeficiente de determinação $\left(\mathrm{R}^{2}\right)$, índice de heterogeneidade do solo (b), tamanho ótimo de parcela (inteiro superior de $\mathrm{X}_{0}$ ), diferença entre médias em porcentagem da média (D), o número de unidades básicas do ensaio (NUB) e de repetições (r) e as dimensões largura (L) e comprimento (C) das áreas dos ensaios em branco, para verificar a associação entre eles. As correlações entre $\mathrm{L}$ e $\mathrm{C}$, e entre $\mathrm{M}_{1}$ (constante em todos os casos) e as demais são nulas e, por isso, foram excluídas. Procedeu-se ao diagnóstico de multicolinearidade na matriz de correlações entre L, C, NUB, b, A, B, R ${ }^{2}, \mathrm{X}_{0}$ e D, com a finalidade de descartar variáveis independentes indesejáveis para a realização da análise de causa e efeito (trilha), tomando $\mathrm{X}_{0}$ e D como variáveis dependentes (Vencovsky \& Barriga, 1992; Cruz \& Regazzi, 1994).

Nos cálculos estatísticos, foram utilizados programas computacionais estatísticos: software científico - NTIA, desenvolvido pela Embrapa Informática Agropecuária (Embrapa, 1997); o programa GENES (Cruz, 2001); o programa SAEG (Ribeiro Júnior, 2001) e um programa em linguagem Fortran (Abou-El-Fittouh et al., 1974), com modificações, específico para calcular a média e as variâncias de parcelas de diferentes tamanhos.

\section{Resultados e Discussão}

Ocorreram variações das estimativas do índice de heterogeneidade do solo, das estimativas dos parâmetros A e $B$ da função $C V(x)=A / X^{B}$, das médias das parcelas de uma unidade básica $\left(\mathrm{M}_{1}\right)$ e dos tamanhos ótimos de parcelas $\left(\mathrm{X}_{0}\right)$ entre os ensaios de cada caso, e variações entre as médias dos diferentes ensaios, tanto dentro de cada caso, como entre ensaios de diferentes casos (Tabela 1). Essas variações entre os ensaios (repetições) foram decorrentes das variações nas condições ambientais do local de cada ensaio. Por sua vez, as variações entre médias de ensaios de diferentes tamanhos foram decorrentes do tamanho do ensaio em largura (L) ou comprimento (C).

Não foram verificadas estimativas médias do índice de heterogeneidade do solo menores que 0,2 que, con- forme interpretação de Lin \& Binns (1986), são casos em que se deve usar maior número de repetições e parcelas de menor tamanho, a fim de maximizar a precisão numa área experimental. Em quatro casos (40\%), o índice de heterogeneidade foi maior do que 0,7 , situação em que a variação no tamanho de parcela é importante para aumentar a precisão experimental.

O tamanho ótimo de parcela $\left(\mathrm{X}_{0}\right)$, estimado pelo método de Meier \& Lessman (1971), depende das estimativas de A e B que, também, são variáveis. Devido à cultura da batata ser cultivada em covas, é necessário usar um valor inteiro para $\mathrm{X}_{0}$. Assim, o tamanho ótimo de parcela, que contempla a maioria dos casos (90\%), foi igual a seis covas, ou seja, uma linha de 1,8 m de comprimento $\left(1,44 \mathrm{~m}^{2}\right)$. Nos casos de se usar o maior valor de $\mathrm{X}_{0}$, entre os ensaios do mesmo caso, pode-se incorrer no erro de superdimensionar o valor de $\mathrm{X}_{0}$ no planejamento dos experimentos, pois, o valor máximo pode ser resultado da falta de uniformidade do terreno, que pode ser facilmente identificado e excluído para a experimentação.

Por sua vez, o uso da média dos $\mathrm{X}_{0}$ estimados a partir de ensaios (repetições), contempla a maioria das situações e, no plano experimental, não será muito diferente do máximo, nos casos em que a amplitude de variação é baixa, porque existe uma compensação do número de repetições possíveis de usar. Também, ao se observar a expressão algébrica para estimar a precisão experimental (D) pelo método de Hatheway (1961), pode-se verificar que pequenas variações nos tamanhos das parcelas podem ser compensadas pelas variações nos números de repetições (r) e de tratamentos (I), e por conseqüência de $t_{1}$ e $t_{2}$, pois o produto $r \times I \times X_{0}$ deve ser menor ou igual ao número de covas (NUB) da área disponível para o experimento. Variações das estimativas de $\mathrm{X}_{0}$, entre repetições e entre genótipos de milho, também foram observadas por Martin et al. (2005), sendo, portanto, consideradas relevantes para o correto planejamento dos experimentos.

Houve variações no número de repetições (r) e na precisão experimental (D), calculada pelo método de Hatheway (1961), nos experimentos com 12 tratamentos, nos dez casos de tamanho de ensaio (Tabela 2). A diminuição da diferença mínima significativa (D), que reduz linearmente com o tamanho da área do ensaio (NUB) (coeficiente de correlação linear entre D e NUB igual a -0,834), é decorrente mais do maior número de repetições (correlação entre $r$ e NUB igual a 0,982), 
que cabem na área, do que do $\mathrm{X}_{0}$ ou das estimativas $\mathrm{A}$, $\mathrm{B}$ e b, que não estão correlacionadas, significativamente, com NUB (Tabela 3). No entanto, quanto maior for a largura do ensaio (L), menores serão as estimativas de $\mathrm{A}, \mathrm{B}$, b e $\mathrm{X}_{0}$. Também, quanto maior o comprimento (C) da área do ensaio, maior a precisão do experimento (menor D). Portanto, experimentos com batata devem ser conduzidos em linhas em que a linha é o bloco, no caso de se usar o delineamento de blocos ao acaso.

A colinearidade da matriz das correlações é intensa (Tabela 3). No entanto, como NUB é proporcional a r e a $L x C, b=2 B$ e $R^{2}$ é uma estatística de qualidade das estimativas, pode-se manter as variáveis A e $\mathrm{B}$ (usadas para estimar $\mathrm{X}_{0}$ ) e, também, $\mathrm{L}$ e C, pelos objetivos do estudo, para a análise de trilha. Neste caso, a colinearidade é moderadamente forte (número de condição Máx/Mín = 360), o que permite realizar a análise de causas e efeitos.

Verifica-se, pelo estudo de causas e efeitos (análises de trilha), que tanto as estimativas de A quanto as de B

Tabela 2. Diferença entre médias de tratamentos em porcentagem da média (D) e número de repetições (r) para experimentos com 12 tratamentos usando a média dos tamanhos de parcela $\left(\mathrm{X}_{0}\right)$ em ensaios com diferente número de covas (NUB) para produtividade de tubérculos de batata.

\begin{tabular}{ccrrc}
\hline Caso & $\mathrm{X}_{0}$ & NUB & $\mathrm{r}$ & $\mathrm{D}$ \\
\hline 1 & 4 & 3.456 & 72 & 11,14 \\
2 & 4 & 1.728 & 36 & 15,78 \\
3 & 6 & 1.728 & 24 & 17,06 \\
4 & 5 & 1.152 & 19 & 20,98 \\
5 & 4 & 864 & 18 & 22,05 \\
6 & 6 & 864 & 12 & 23,67 \\
7 & 6 & 576 & 8 & 27,23 \\
8 & 4 & 576 & 12 & 27,24 \\
9 & 7 & 432 & 5 & 32,41 \\
10 & 5 & 288 & 4 & 41,91 \\
\hline
\end{tabular}

atuam sobre a variação existente com $\mathrm{X}_{0}$ (Tabela 4), pois a correlação entre A e $X_{0}(r=0,961)$ e entre B e $X_{0}$ $(r=0,969)$ (Tabela 3$)$ são decorrentes do efeito direto de $\mathrm{A}$ e indireto de $\mathrm{B}$, ou direto de $\mathrm{B}$ e indireto de $\mathrm{A}$, aproximadamente nas mesmas proporções (Tabela 4).

A correlação entre $\mathrm{X}_{0}$ e $\mathrm{L}(\mathrm{r}=-0,862)$ é decorrente dos efeitos indiretos de $\mathrm{A}$ e $\mathrm{B}$. Como $\mathrm{X}_{0}$ não se correlacionou com $\mathrm{C}$, não faz sentido estudar os efeitos indiretos das outras variáveis sobre $\mathrm{X}_{0}$. Por sua vez, a precisão (D) se correlacionou significativamente com o comprimento (C) do ensaio e os efeitos indiretos de L, A e B podem ser desprezados. Como conseqüência, a execução de experimentos com batata, no delineamento blocos ao acaso, aumenta a precisão do experimento. Neste plano experimental, os blocos devem ser constituídos pelas linhas e as parcelas, constituídas por seis covas, e devem ser posicionados dentro da mesma linha. Com o uso deste bloqueamento, existe um ganho em precisão, não estimado, pela eliminação da variação entre as linhas. No caso de experimento com 12 tratamentos, seriam necessárias 72 covas (12x6) por linha (bloco) e o número de linhas ou número de blocos (r) dependeria da precisão (D), previamente determinada e estimada pela expressão $r=2\left(t_{1}+t_{2}\right)^{2} A^{2} / D^{2} X_{0}^{b}$ de Hatheway (1961).

Os resultados indicam que o uso de áreas experimentais com maior número de covas por linha (maior C) permite planejar experimentos mais precisos (menor D), usando seis covas por parcela e ajustando o número de repetições e o de tratamentos para adequar a área disponível. Grande parte das causas de variação observadas devem ser devidas ao tipo de manejo usado na cultura, pois, a adubação, plantio e amontoa é mecanizada e realizada no sentido das linhas, conforme recomendações técnicas da cultura (Bisognin, 1996).

Tabela 3. Coeficientes de correlação linear simples entre estimativas dos parâmetros da função $\mathrm{CV}(\mathrm{x})=\mathrm{A} / \mathrm{X}^{\mathrm{B}}$, coeficiente de determinação $\left(\mathrm{R}^{2}\right)$, índice de heterogeneidade do solo (b), tamanho ótimo de parcela $\left(\mathrm{X}_{0}\right)$, diferença entre médias em porcentagem da média (D), número de unidades básicas do ensaio (NUB) e de repetições (r) e dimensões largura (L) e comprimento (C) das áreas dos ensaios em branco.

\begin{tabular}{|c|c|c|c|c|c|c|c|c|}
\hline & NUB & $\mathrm{b}$ & A & B & $\mathrm{R}^{2}$ & $\mathrm{X}_{0}$ & $\mathrm{D}$ & $\mathrm{r}$ \\
\hline $\mathrm{L}$ & 0,433 & $-0,896^{*}$ & $-0,800^{*}$ & $-0,897^{*}$ & $0,592^{*}$ & $-0,862 *$ & $-0,534$ & 0,539 \\
\hline C & $0,855^{*}$ & $-0,095$ & 0,035 & $-0,952 *$ & 0,354 & $-0,011$ & $-0,748^{*}$ & $0,751 *$ \\
\hline NUB & & $-0,484$ & $-0,364$ & $-0,484$ & $0,634 *$ & $-0,401$ & $-0,834 *$ & $0,982 *$ \\
\hline b & & & $0,964 *$ & $0,999 *$ & $-0,526$ & $0,969^{*}$ & 0,493 & $-0,583$ \\
\hline A & & & & $0,964 *$ & $-0,395$ & $0,961^{*}$ & 0,311 & $-0,482$ \\
\hline B & & & & & $-0,526$ & $0,969^{*}$ & 0,494 & $-0,583$ \\
\hline $\mathrm{R}^{2}$ & & & & & & $-0,431$ & $-0,539$ & $0,661 *$ \\
\hline $\mathrm{X}_{0}$ & & & & & & & 0,372 & $-0,521$ \\
\hline D & & & & & & & & $-0,798^{*}$ \\
\hline
\end{tabular}

*Significativo pelo teste t a 5\% de probabilidade, com 8 graus de liberdade. 
Tabela 4. Efeitos diretos e indiretos das estimativas médias dos parâmetros da função $\mathrm{CV}(\mathrm{x})=\mathrm{A} / \mathrm{X}^{\mathrm{B}}$ e das dimensões da largura (L) e comprimento (C) sobre a estimativa do tamanho ótimo de parcela $\left(\mathrm{X}_{0}\right)$ e sobre a diferença entre médias em porcentagem da média (D).

\begin{tabular}{clrr}
\hline Parâmetro & \multicolumn{1}{c}{ Efeito } & $\mathrm{X}_{0}$ & $\mathrm{D}$ \\
\hline $\mathrm{A}$ & Direto sobre & 0,4440 & $-0,5971$ \\
& Indireto via B & 0,4342 & 0,4701 \\
& Indireto via L & 0,0824 & 0,4615 \\
& Indireto via C & 0,0006 & $-0,0236$ \\
& Total & 0,9613 & 0,3109 \\
\hline $\mathrm{B}$ & Direto sobre & 0,4503 & 0,4875 \\
& Indireto via A & 0,4282 & $-0,5758$ \\
& Indireto via L & 0,0924 & 0,5172 \\
& Indireto via C & $-0,0015$ & 0,0648 \\
& Total & 0,9694 & 0,4937 \\
\hline $\mathrm{L}$ & Direto sobre & $-0,1031$ & $-0,5769$ \\
& Indireto via A & $-0,3552$ & 0,4777 \\
& Indireto via B & $-0,4037$ & $-0,4371$ \\
& Indireto via C & 0,0000 & 0,0000 \\
& Total & $-0,8620$ & $-0,5363$ \\
\hline $\mathrm{C}$ & Direto sobre & 0,0166 & $-0,6808$ \\
& Indireto via A & 0,0154 & $-0,0207$ \\
& Indireto via B & $-0,0428$ & $-0,0464$ \\
& Indireto via L & 0,0000 & 0,0000 \\
& Total & $-0,0107$ & $-0,7479$ \\
\hline
\end{tabular}

Como conseqüência, os dados de parcelas experimentais de batata (ensaios pequenos) podem ser usados para estimar o índice de heterogeneidade do solo e o tamanho ótimo de parcela, visando à identificação de fatores (tratamentos) que influenciam o planejamento experimental, como o procedido por Martin et al. (2005) com a cultura do milho e Lopes et al. (2005) com a cultura do sorgo. Não é necessária a instalação de ensaios específicos, como o procedido por Oliveira \& Estefanel (1995), para estimativas do tamanho de parcela em batata, podendo-se usar os dados de experimentos, desde que a colheita tenha sido realizada em parcelas pequenas (covas) ou em algumas áreas menores e representativas dentro de área maior, a qual será usada para o experimento.

\section{Conclusões}

1. O tamanho dos ensaios com a cultura de batata, medido em número de covas, não influencia a estimativa do índice de heterogeneidade do solo e a estimativa do tamanho ótimo de parcela.

2. O uso de áreas experimentais com maior número de covas por linha permite planejar experimentos mais precisos para a avaliação da produtividade de tubérculos de batata.
3. O uso de parcelas com seis covas na linha e a adoção da linha como bloco aumenta a precisão dos experimentos com a cultura da batata.

\section{Referências}

ABOU-EL-FITTOUH, H.A.; EL-BAKRY, A.E.; EL-SERGANY, D.Z. A program for studying the optimum plot size in field experiments on the computer. Agricultural Research Review, v.52, p.85-90, 1974.

BAKKE, O.A. Tamanho e forma de parcelas em delineamentos experimentais. 1988. 142p. Dissertação (Mestrado) - Escola Superior de Agricultura Luiz de Queiroz, Piracicaba.

BISOGNIN, D.A. (Coord.). Recomendações técnicas para o cultivo da batata no Rio Grande do Sul e Santa Catarina. Santa Maria: UFSM, 1996. 64p.

CRUZ, C.D. Programa GENES: versão Windows; aplicativo computacional em genética e estatística. Viçosa: UFV, 2001. 648p.

CRUZ, C.D.; REGAZZI, A.J. Modelos biométricos aplicados ao melhoramento genético. Viçosa: UFV, 1994. 390p.

EMBRAPA. Centro Nacional de Pesquisa Tecnológica em Informática para a Agricultura (Campinas, SP). Ambiente de software NTIA: versão 4.2.2: manual do usuário - ferramental estatístico. Campinas, 1997. 258p.

GOMEZ, K.A.; GOMEZ, A.A. Statistical procedures for agricultural research. $2^{\text {nd }}$ ed. New York: Wiley, 1984. 680p.

HATHEWAY, W.H. Convenient plot size. Agronomy Journal, v.53, p.279-280, 1961.

LESSMAN, K.J.; ATKINS, R.E. Comparisons of planning arrangements and estimates of optimum hill plot for grain sorghum yield tests. Crop Science, v.3, p.477-481, 1963.

LIN, C.S.; BINNS, M.R. Relative efficiency of two randomized block designs having different plot size and numbers of replications and of plots per block. Agronomy Journal, v.78, p.531-534, 1986. LOPES, S.J.; STORCK, L. A precisão experimental para diferentes manejos na cultura do milho. Ciência Rural, v.25, p.49-53, 1995.

LOPES, S.J.; STORCK, L.; GARCIA, D.C. A precisão de ensaios de cultivares sob diferentes adubações. Ciência Rural, v.24, p.483487, 1994.

LOPES, S.J.; STORCK, L.; LÚCIO, A.D.; LORENTZ, L.H.; LOVATO, C.; DIAS, V.O. Tamanho de parcela para produtividade de grãos de sorgo granífero em diferentes densidades de plantas. Pesquisa Agropecuária Brasileira, v.40, p.525-530, 2005.

MARTIN, T.N.; STORCK, L.; LÚCIO, A.D.; CARVALHO, M.P.; SANTOS, P.M. Bases genéticas de milho e alterações no plano experimental. Pesquisa Agropecuária Brasileira, v.40, p.35-40, 2005.

MEIER, V.D.; LESSMAN, K.J. Estimation of optimum field plot shape and size testing yield in Crambe abyssinica Hordnt. Crop Science, v.11, p.648-650, 1971. 
OLIVEIRA, P.H.; ESTEFANEL, V. Tamanho e forma ótimos da parcela para avaliação do rendimento em experimentos com batata. Ciência Rural, v.25, p.205-208, 1995.

RAMALHO, M.A.P.; FERREIRA, D.F.; OLIVEIRA, A.C. de. A experimentação em genética e melhoramento de plantas. Lavras: Edufla, 2000. 326p.

RIBEIRO JÚNIOR, J.I. Análises estatísticas no SAEG. Viçosa: UFV, 2001. 301p.

SMITH, H.F. An empirical law describing heterogeneity in the yields of agricultural crops. Journal of Agricultural Science, v.28, p.123, 1938.
STEEL, R.G.D.; TORRIE, J.H.; DICKEY, D.A. Principles and procedures of statistics: a biometrical approach. $3^{\text {rd }}$ ed. New York: McGraw Hill Book, 1997. 666p.

STORCK, L.; GARCIA, D.C.; LOPES, S.J.; ESTEFANEL, V. Experimentação vegetal. Santa Maria: UFSM, 2000. 198p.

THOMAS, E.J. Relationship between plot size and plot variance. Agricultural Research Journal of Kerala, v.12, p.178-189, 1974.

VENCOVSKY, R.; BARRIGA, P. Genética biométrica no fitomelhoramento. Ribeirão Preto: Revista Brasileira de Genética, 1992. 496p.

Recebido em 11 de março de 2005 e aprovado em 3 de março de 2006 